Eur. J. Clin. Chem. Clin. Biochem.

Vol. 30, 1992, pp. 381-385

(C) 1992 Walter de Gruyter \& Co.

Berlin · New York

\title{
Beeinflussung membrangebundener Enzymaktivitäten durch Veränderung der Membranlipidzusammensetzung
}

\author{
Von Bärbel Boßmann und H. J. Hütter \\ Institut für Pathologische Biochemie der Martin-Luther-Universität Halle-Wittenberg, Halle/Saale
}

(Eingegangen am 12. März 1991/26. März 1992)

Zusammenfassung: Nach zweieinhalbstündiger in vivo-Perfusion des Rattenjejunums mit wäßrigem Ethanol (Volumenanteil 0,05) bzw. Tetrachlorkohlenstoff (Volumenanteil 0,005) wurden in den präparierten Membranvesikeln des Bürstensaums deutlich verminderte Cholesterin- und Phospholipidkonzentrationen nachgewiesen. Ihnen entsprachen Aktivitätsverluste membrangebundener Enzyme. Tetrachlorkohlenstoff beeinflußte die Membranen stärker als Ethanol. Als Ursache werden physikochemische Veränderungen in den hydrophoben Membranregionen an den Verankerungen der Enzyme diskutiert.

Modification of membrane lipid composition by lipophilic agents, and its effect on the activity of membranebound enzymes

Summary: Rat jejunum was perfused for two and a half hours with aqueous ethanol (volume fraction 0.05) or aqueous carbon tetrachloride (volume fraction 0.005). Membrane vesicles subsequently prepared from the brush border displayed markedly decreased contents of cholesterol and phospholipids, as well as decreases in the activities of membrane-bound enzymes. These decreases were more pronounced in vesicles prepared after carbon tetrachloride than after ethanol perfusion. It is suggested that the anchoring of membrane-bound enzymes is decreased by physical chemical alterations in the hydrophobic region of the membrane.

\section{Einführung}

Intestinale apikale Bürstensaummembranen bestehen aus Proteinen und Lipiden, die als Doppelschicht die Grundstruktur bilden. Der Proteinanteil umfaßt zwei Gruppen von Membranproteinen, die peripher (extrinsisch) den Membranen aufliegen, oder integral (intrinsisch) unter hydrophoben Wechselwirkungen in die Membranlipide eingebettet sind und für ihre Funktionen als Rezeptoren, Transportproteine und Enzyme amphipolare Lipide als Umgebung benötigen. Jede Alteration der Membranzusammensetzung verändert ihre Permeabilitäts-, Transport- und Enzymfunktionen $(1-6)$. Zum Verhalten integraler Membranenzyme existieren hierzu nur Hinweise über alkalische Phosphatase $(2,7)$, Adenylatcyclase (3) und Acyl-CoA-Cholesterol-Acyltransferase (8).

Die Enzyme des Peptidstoffwechsels Alaninaminopeptidase, $\gamma$-Glutamyltransferase und Dipeptidylpeptidase IV sind integrale Membranproteine wie zwei bisher noch nicht klassifizierte Enzyme, eine Dipeptidase (9) und eine Aminopeptidase (10). Zusätzlich haben wir die Aktivität der ebenso lokalisierten alkalischen Phosphatase bestimmt.

Ziel unserer Untersuchungen war das Verhalten der membrangebundenen Enzymaktivitäten nach Modifizierung der intestinalen Bürstensaummembranen durch lipophile Verbindungen. 


\section{Material und Methoden}

Die Perfusionsversuche wurden an narkotisierten (Urethan 200 $\mathrm{g} / \mathrm{l} ; 1 \mathrm{ml}$ ) weiblichen weißen Wistarratten von 200 bis $250 \mathrm{~g}$ Körpergewicht vorgenommen. Dazu wurden etwa $20 \mathrm{~cm}$ proximales Jejunum freigelegt und dieses Segment über zu- und abführenden Katheter antegrad mit temperierter Ringer-Lösung mit lipophilem Agens (Ethanol, Volumenanteil 0,05 bzw. Tetrachlorkohlenstoff, Volumenanteil 0,005) 2,5 Stunden in vivo durchströmt. Nach der Perfusion präparierten wir aus der abgeschabten Mucosa die Bürstensaummembran-Vesikel nach Schmitz et al. (11). Der Anreicherungseffekt wurde über die Bestimmung der Membranenzyme kontrolliert.

Als Kontrollen dienten Membranvesikel von jejunalen Segmenten unbehandelter Tiere. Wir untersuchten die Enzyme in den Perfusaten und in den Vesikelpräparationen (Tab. 1).

Die Ergebnisse wurden mit dem verteilungsfreien $\chi^{2}$-Test und einer Irrtumswahrscheinlichkeit von $\mathrm{p}<0,01 \quad(* *)$ und $\mathrm{p}<0,05(*)$ ausgewertet.

\section{Ergebnisse}

Das Verhalten der Lipide und Enzymaktivitäten in den Membranvesikeln nach Einwirkung der lipophilen Verbindungen ist in den Tabellen 2 bis 4 dargestellt. Unter Tetrachlorkohlenstoff fallen Lipid- und Enzymaktivitätskonzentrationen stärker ab als unter Ethanol. Dabei vermindert Tetrachlorkohlenstoff den Cholesteringehalt stärker als die Konzentration der Phospholipide. Er erniedrigt außerdem den Proteingehalt der Vesikel wesentlich. Dadurch nehmen die spezifischen Aktivitäten gegenüber denen der Kontrollen kaum ab, obwohl die Substratumsätze erniedrigt sind. Ethanolperfusion vermindert auch die spezifischen Aktivitäten.
Tab. 1. Übersicht der angewandten analytischen Methoden

Analyt und Methode (Literatur)

- Protein nach Lowry et al. (modifiziert nach Glässer \& Kleine (12))

- Cholesterin nach Zak (13)

- Phospholipide nach Bartlett (14)

- Alaninaminopeptidase/microsomal aminopeptidase, EC 3.4.11.2, nach Farr et al. (15)

- Alkalische Phosphatase/Orthophosphoric-monoester Phosphohydrolase, alkaline optimum, EC 3.1.3.1. nach Bernt (16)

- $\gamma$-Glutamyltransferase, EC 2.3.2.2, nach Szász (17)

- Dipeptidylpeptidase IV, EC 3.4.14.5, nach Küllertz et al. (18)

- C-terminale konfigurationsunabhängige Dipeptidase, bisher nicht klassifiziert, Gly-D-Leu als Substrat, nach Campbell (9)

- Leucinhydrazidhydrolase, nicht klassifiziert, Leu- $\mathrm{NHNH}_{2}$ als Substrat, nach Kenny et al. (10)

- Leucinaminopeptidase/Aminopeptidase cytosolisch, EC 3.4.11.1, nach Haschen et al. (19)

- $\beta$-Glucuronidase/ $\beta$ - $D$-glucuronoside glucuronosohydrolase, EC 3.2.1.31, nach Szász (20)

- Hydrolyse von Di- und Tripeptiden, Nachweis papierchromatographisch nach Hanson (21)

Die Fähigkeit zum Umsatz von Di- und Tripeptiden ist im Bereich von Bürstensaummembranen neben der Alaninaminopeptidase (22) noch einer Dipeptidase (bevorzugtes Substrat: Gly-D-Leu) zuzuordnen (9).

Tab. 2. Cholesterin- und Phospholipidgehalt in jejunalen Bürstensaummembran-Vesikeln nach Einwirkung lipophiler Substanzen

\begin{tabular}{|c|c|c|c|c|c|c|}
\hline & \multicolumn{6}{|c|}{ Vesikelpräparation } \\
\hline & \multicolumn{2}{|c|}{$\begin{array}{l}\text { aus unbehandeltem } \\
\text { Dünndarm }(\mathrm{n}=8)\end{array}$} & \multicolumn{4}{|c|}{ nach Perfusion mit } \\
\hline & \multirow[b]{2}{*}{$\overline{\mathrm{x}}$} & \multirow[b]{2}{*}{ $\pm s_{\bar{x}}$} & \multicolumn{2}{|c|}{$\begin{array}{l}\text { Tetrachlor- } \\
\text { kohlenstoff }(n=5) \\
\text { Volumenanteil } 0,005\end{array}$} & \multicolumn{2}{|c|}{$\begin{array}{l}\text { Ethanol }(\mathrm{n}=5) \\
\text { Volumenanteil } 0,05\end{array}$} \\
\hline & & & $\overline{\mathrm{x}}$ & $\pm s_{\overline{\mathbf{x}}}$ & $\overline{\mathbf{x}}$ & $\pm s_{\bar{x}}$ \\
\hline $\begin{array}{l}\text { Cholesterin } \\
{[\mu \mathrm{mol} / 1]}\end{array}$ & 201,0 & 12,5 & $76,5^{*}$ & 8,0 & $145,5^{*}$ & 9,5 \\
\hline $\begin{array}{l}\text { Cholesterin } \\
\text { [nmol/mg Protein] }\end{array}$ & 309,5 & & 186,5 & & 220,5 & \\
\hline $\begin{array}{l}\text { Phospholipide } \\
{[\mu \mathrm{mol} / 1]}\end{array}$ & 291,0 & 27,1 & $168,0^{*}$ & 9,2 & $210,0^{*}$ & 19,1 \\
\hline $\begin{array}{l}\text { Phospholipide } \\
\text { [nmol/mg Protein] }\end{array}$ & 450,0 & & 416,0 & & 319,0 & \\
\hline $\begin{array}{l}\text { Cholesterin/Phospholipide } \\
{[\mathrm{mol} / \mathrm{mol}]}\end{array}$ & 0,690 & & 0,450 & & 0,690 & \\
\hline
\end{tabular}

$* \mathrm{p}<0,05$ im Vergleich zur Kontrolle 


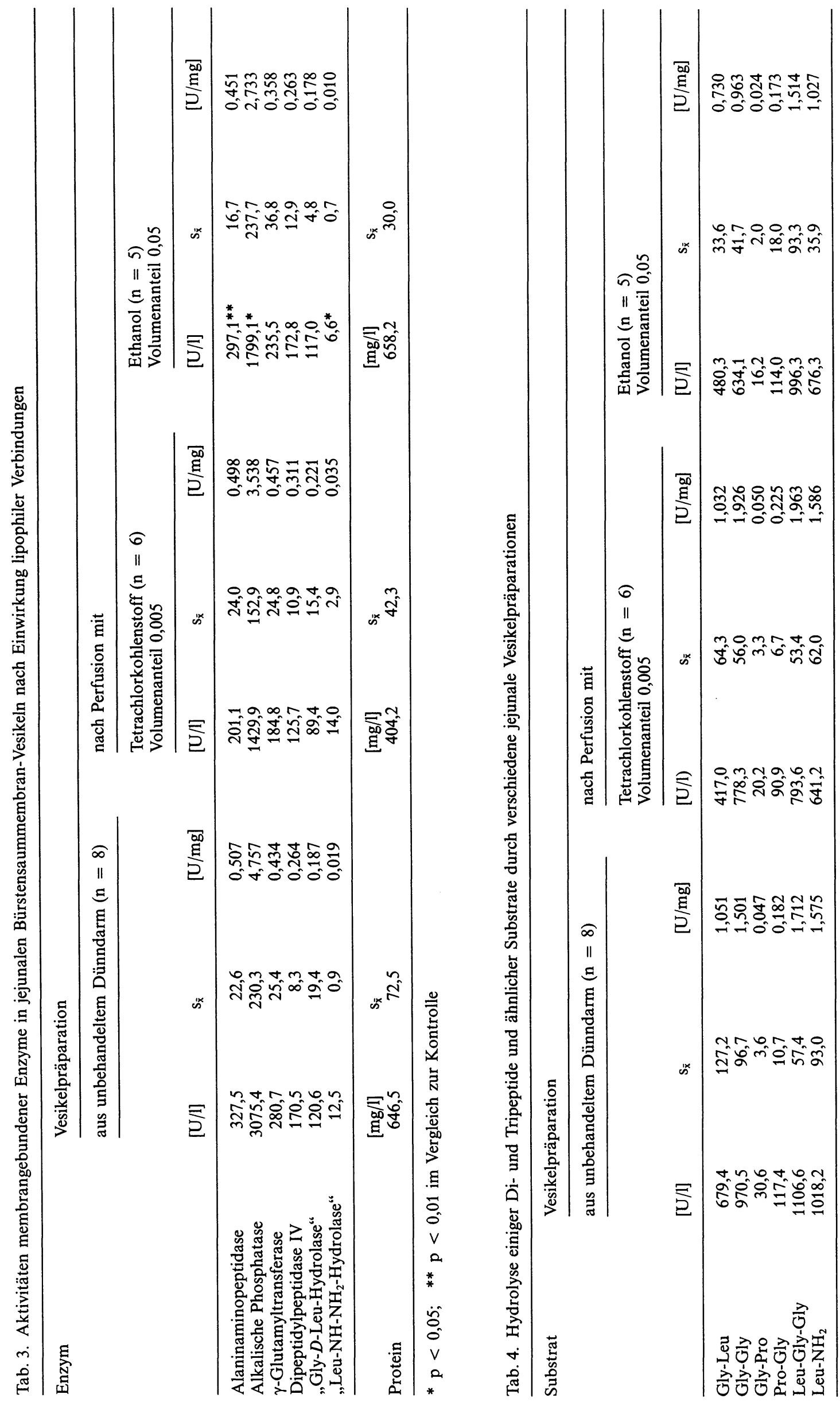


Beide Lösungsmittel vermindern diese Umsätze ebenfalls deutlich, wobei wegen des verminderten Proteingehalts unter Tetrachlorkohlenstoff die spezifischen Aktivitäten wiederum weniger markant als unter Ethanol abfallen.

In vitro-Inkubationen von Membranvesikeln unbehandelter Tiere mit der entsprechenden Menge Ethanol bzw. Tetrachlorkohlenstoff hemmten Alaninaminopeptidase, $\gamma$-Glutamyltransferase und alkalische Phosphatase um 5 bis $20 \%$.

Um zu klären, ob während der in vivo-Perfusionen durch den Kontakt der Mucosa mit den eingesetzten Substanzen bereits eine Membranschädigung auftritt, haben wir Enzyme von lysosomaler und cytosolischer Lokalisation im Perfusat untersucht und mit Ergebnissen nach Chenodesoxycholat-Einwirkung (23) in Beziehung gesetzt. Die in Tabelle 5 angegebenen hohen Aktivitäten nach Chenodesoxycholat korrelierten mit vereinzelten Zelläsionen, die elektronenmikroskopisch nachgewiesen wurden.

\section{Diskussion}

Unsere heutigen Vorstellungen über Biomembranen basieren auf dem „Fluid-Mosaic"-Modell von Singer \& Nicolson (24), nach dem eine Lipiddoppelschicht das Gerüst einer Membran bildet, in der Proteine iateral frei beweglich sind.

Wir haben als Modell für die erwarteten Wechselwirkungen zwischen Biomembranen und lipophilen Verbindungen Bürstensaummembran-Vesikel eingesetzt, für deren Membranbereiche keine Primärschädigung während der in vivo-Perfusionen anzunehmen ist (Tab. 5).
Fluiditätsänderungen werden immer auch die laterale Beweglichkeit der Membranproteine beeinflussen und damit die Membranfunktionen verändern. Fluiditätsanstiege wurden nach Erhöhung des Gehalts an ungesättigten Fettsäuren, Fluiditätsabnahmen nach Erhöhung der Cholesterinkonzentration in der Membran beschrieben $(2,3)$. Nach Taraschi \& Rubin (25) führt kurzfristige Alkoholgabe zur Einlagerung in Biomembranen mit Fluiditätszunahme (disordering effect). Lewis et al. (26) bestätigen dieses an Modellbilayer-Systemen mit niedriger Ethanolkonzentration und beschreiben bei höheren Konzentrationen Fluiditätsverluste.

Auch Tetrachlorkohlenstoff verändert die Lipidzusammensetzung von Biomembranen. Bisher fehlen Untersuchungen an membranständigen sowie membranintegrierten Enzymen des Peptidstoffwechsels über die möglichen Auswirkungen von Fluiditätsänderungen auf Membranfunktionen.

Dipeptidylpeptidase IV und $\gamma$-Glutamyltransferase besitzen eine hohe Substratspezifität, für die Dipeptidase wird der Umsatz vieler Dipeptide und von Leukotrien D4 beschrieben (9). Die Alaninaminopeptidase ist zwar als Aminopeptidase mit bevorzugtem Umsatz von Aminosäureamiden klassifiziert, hydrolysiert aber auch Di-, Tri- und höhermolekulare Peptidverbindungen, wie die Hormone Oxytocin und Vasopressin (27). Nach unseren Untersuchungen verändert sich die Aktivität sowohl von Peptidasen mit hoher als auch von solchen mit geringer Substratspezifität unter den von uns gewählten Versuchsbedingungen. Da es sich bei allen Enzymen um integrale Membranproteine handelt, die über Peptidstrukturen

Tab. 5. Cytosolische und lysosomale Enzymaktivitäten in den Perfusaten

\begin{tabular}{|c|c|c|c|c|}
\hline \multirow[t]{2}{*}{ Enzym } & & \multicolumn{3}{|c|}{ Ringer-Perfusat unter Zusatz von } \\
\hline & & $\begin{array}{l}\text { Tetrachlorkohlenstoff } \\
\text { Volumenanteil } 0,005 \\
\text { [mU/Vol.] }\end{array}$ & $\begin{array}{l}\text { Ethanol } \\
\text { Volumenanteil } 0,05 \\
\text { [mU/Vol.] }\end{array}$ & $\begin{array}{l}\text { Chenodesoxycholat* } \\
3 \mathrm{mmol} / 1 \\
\text { [mU/Vol.] }\end{array}$ \\
\hline Leucinaminopeptidase & $\begin{array}{l}\overline{\mathrm{x}} \\
\mathrm{s}\end{array}$ & $\begin{array}{l}390 \\
160\end{array}$ & $\begin{array}{l}74 \\
37\end{array}$ & $\begin{array}{l}5440 \\
1200\end{array}$ \\
\hline Gly-Leu-Hydrolyse & $\begin{array}{l}\bar{x} \\
s\end{array}$ & $\begin{array}{r}2520 \\
870\end{array}$ & $\begin{array}{r}1220 \\
360\end{array}$ & $\begin{array}{l}9470 \\
1830\end{array}$ \\
\hline Gly-Pro-Hydrolyse & $\begin{array}{l}\overline{\mathrm{x}} \\
\mathrm{s}\end{array}$ & $\begin{array}{l}2560 \\
1030\end{array}$ & $\begin{array}{l}950 \\
400\end{array}$ & $\begin{array}{r}10800 \\
1240\end{array}$ \\
\hline$\beta$-Glucuronidase & $\begin{array}{l}\overline{\mathrm{x}} \\
\mathrm{s}\end{array}$ & $\begin{array}{l}0,42 \\
0,71\end{array}$ & $\begin{array}{l}0 \\
0\end{array}$ & $\begin{array}{r}10,1 \\
1,6\end{array}$ \\
\hline
\end{tabular}

* siehe 1.c. (23) 
vorwiegend hydrophober Aminosäuren in der Lipiddoppelschicht verankert sind, halten wir eine direkte Wechselwirkung der eingesetzten lipophilen Verbindungen mit dieser Region für möglich.

\section{Literatur}

1. Brasitus, T. A. \& Schachter, D. (1982) Cholesterol biosynthesis and modulation of membrane cholesterol and lipid dynamics in rat intestinal microvillus membranes. Biochemistry $21,2241-2246$.

2. Brasitus, T. A., Davidson, N. O. \& Schachter, D. (1985) Variations in dietary triacylglycerol saturation alter the lipid composition and fluidity of rat intestinal plasma membranes. Biochim. Biophys. Acta 812, 460-472.

3. Hagve, T. A. (1988) Effects of unsaturated fatty acids on cell membrane functions. Scand. J. Clin. Lab. Invest. 48, $381-388$.

4. Meddings, J. B. (1988) Lipid permeability of rat jejunum and ileum: correlation with physical properties of the microvillus membrane. Biochim. Biophys. Acta 943, 305-314.

5. Merill, A. R., Proulx, P. \& Szabo, A. (1986) Effects of exogenous fatty acids on calcium uptake by brush-border membrane vesicles from rabbit small intestine. Biochim. Biophys. Acta $855,337-344$.

6. Schedl, H. P., Wilson, H. D., Mathur, S. N., Murthy, S. \& Field, F. J. (1989) Effects of phospholipid or cholesterol enrichment on rat intestinal brush border membrane order and transport of calcium. Metabolism 38, 1164-1169.

7. Brasitus, T. A., Dahiya, R., Dudeja, P. K. \& Bissonnette, B. M. (1988) Cholesterol modulates alkaline phosphatase activity of rat intestinal microvillus membranes. J. Biol. Chem. 263, 8592-8597.

8. Brenneman, D. E., Kaduce, T. \& Spector, A. A. (1977) Effect of dietary fat saturation on acylcoenzyme A: cholesterol acyltransferase activity of Ehrlich cell microsomes. J. Lipid Res. 18, 582-591.

9. Campbell, B. J. (1970) Renal dipeptidase. Methods Enzymol. 19, $722-729$.

10. Kenny, A. J., Booth, A. G. \& MacNair, R. D. C. (1977) Peptidases of the kidney microvillus membrane. Acta Biol. Med. Germ. 36, 1575-1585.

11. Schmitz, J., Preiser, H., Maestracci, D., Ghosh, B. K., Cerda, J. J. \& Crane, R. K. (1973) Purification of the human intestinal brush border membrane. Biochim. Biophys. Acta 323, 98-112.

12. Glässer, D. \& Kleine, R. (1962) Beiträge zur Eiweißbestimmung in stark verdünnten Lösungen. Pharmazie 17, 3236.

13. Zak, B. (1977) Cholesterol methodologies: A review. Clin. Chem. 23, $1201-1214$.

14. Bartlett, G. R. (1959) Phosphorus assay in column chromatography. J. Biol. Chem. 234, 466-468.
Die auf Basis der von uns ermittelten geänderten Lipidzusammensetzung und aus vorliegenden Literaturbefunden gezogenen Rückschlüsse auf einen Fluiditätsanstieg sind jedoch noch durch direkte Fluiditätsmessungen zu belegen.

15. Farr, W., Rehfeld, N., Reichelt, D. \& Haschen, R. J. (1968) Vergleichende Untersuchungen zur Bestimmung der Aminosäurearylamidase im menschlichen Serum. Z. Med. Labortechn. 9, 78-86.

16. Bernt, E. (1970) Alkalische Phosphatase in Milch. Methoden der enzymatischen Analyse (Bergmeyer, H. U., Hrsg.) 2. Auflage, Band I, pp. 830-832 Akademie-Verlag Berlin.

17. Szasz, G. (1969) A kinetic photometric method for serum $\gamma$-glutamyl transpeptidase. Clin. Chem. 15, 124-136.

18. Küllertz, G., Fischer, G. \& Schönberner, H. (1982) Bestimmung und physiologische Aspekte der DP IV-Exkretion in den humanen Urin. Wissenschaftliche Beiträge der MartinLuther-Universität Halle/Wittenberg 48 (R 75), 175-179.

19. Haschen, R. J., Farr, W. \& Reichelt, D. (1968) Photometrische Bestimmung der „klassischen“ Leucinaminopeptidase im Blutplasma und -serum. Z. Klin. Chem. Klin. Biochem. 6, 11-18.

20. Szasz, G. (1967) Die Bestimmung der $\beta$-GlucuronidaseAktivität im Serum mit p-Nitrophenyl-glucuronid. Clin. Chim. Acta 15, 275-282.

21. Hanson, H. (1966) Peptidasen (Exopeptidasen); in HoppeSeyler/Thierfelder, Handbuch der physiologisch- und pathologisch-chemischen Analyse, Band 6 Teil C, pp. 40-43, Springer-Verlag, Berlin-Heidelberg-New York.

22. Hütter, H. J., Ugolev, A. M., Egorova, V. V. \& Nikitina, A. A. (1986) Alaninaminopeptidasen aus Darmschleimhaut von Hund, Kaninchen und Forelle. I. Charakterisierung der Protease-Formen. Biomed. Biochem. Acta 45, 845853.

23. Boßmann, B. \& Haschen, R. J. (1983) Release of enzymes from rat jejunal mucosa by bile salts. J. Clin. Chem. Clin. Biochem. 21, 1-9.

24. Singer, S. J. \& Nicolson, G. L. (1972) The fluid mosaic model of the structure of cell membranes. Science 175 , $720-731$.

25. Taraschi, T. F. \& Rubin, E. (1985) Biology of disease: Effects of ethanol on the chemical and structural properties of biologic membranes. Lab. Invest. 52, 120-131.

26. Lewis, E. N., Levin, I. W. \& Steer, C. J. (1989) Infrared spectroscopic study of ethanol-induced changes in rat liver plasma membrane. Biochim. Biophys. Acta 986, 161-166.

27. Hütter, H. J., Barth, T. \& Pliska, V. (1970) Hydrolysis of [Asn,Val]-angiotensin II by kidney aminopeptidases. Endocrinologia Experimentalis 4, 19-23.

Dr. rer. nat. B. Boßmann

Institut für Pathologische Biochemie

Martin-Luther-Universität

Magdeburger Straße 2

O-4020 Halle/Saale 
